\title{
Trivalent Chromium Modulates Hexosamine Biosynthesis Pathway Transcriptional Activation of Cholesterol Synthesis and Insulin Resistance
}

\author{
Brent A. Penque, Lixuan Tackett, Jeffrey S. Elmendorf \\ Departments of Cellular and Integrative Physiology and Biochemistry and Molecular Biology, Centers for Diabetes Research, and \\ Membrane Biosciences, Indiana University School of Medicine, Indianapolis, USA \\ Email: jelmendo@iupui.edu
}

Received April 23, 2013; revised May 25, 2013; accepted June 26, 2013

Copyright (C) 2013 Brent A. Penque et al. This is an open access article distributed under the Creative Commons Attribution License, which permits unrestricted use, distribution, and reproduction in any medium, provided the original work is properly cited.

\begin{abstract}
Trivalent chromium has long been recognized to benefit carbohydrate and lipid metabolism. Given emerging evidence that suggests chromium improves insulin sensitivity through the maintenance of an optimal level of plasma membrane (PM) cholesterol, we delineated the role of this micronutrient in attenuating hyperinsulinemia-induced cholesterol biosynthesis and insulin resistance. Exposing 3T3-L1 adipocytes to physiological hyperinsulinemia (500 pM $12 \mathrm{~h}$ ), resulted in a marked impairment in insulin-stimulated glucose transport. Concurrent treatment with chromium in the picolinate form (CrPic, $10 \mathrm{nM} 16 \mathrm{~h}$ ) prevented against glucose transport dysfunction. Insulin signaling was neither impaired by hyperinsulinemia nor amplified by chromium to promote this protective action. Instead, it was found that hyperinsulinemia promoted an increase in PM cholesterol content that was observed to impair the acute ability of insulin to stimulate GLUT4 redistribution to the PM. Chromium prevented against the accumulation of PM cholesterol. Mechanistically, hyperinsulinemia promoted increases in $O$-GlcNAc modification of specificity protein 1 (Sp1), known to engage a cholesterolgenic response. Subsequent chromatin immunoprecipitation and luciferase assays revealed that hyperinsulinemia increased the binding affinity of Sp1 to the promoter region of Hmgcr, encoding 3-hydroxy 3-methyl-glutaryl-CoA reductase (HMGR), as well as HMGR promoter activity. This resulted in gains in mRNA and protein content of HMGR, with resulting elevations in PM cholesterol content. Moreover, treatment with chromium prevented this transcriptional response. Together, these data suggest a mechanism whereby CrPic affords glycemic health through inhibition of a transcriptional cholesterolgenic program detrimental to insulin action.
\end{abstract}

Keywords: 3T3-L1 Adipocytes; GLUT4; HMG-CoA Reductase; Hyperinsulinemia; Sp1

\section{Introduction}

Clinical trials have revealed beneficial actions of trivalent chromium $\left(\mathrm{Cr}^{3+}\right)$ on glycemic control [1-3], yet a mechanism of action remains unknown. In this regard, data collected in our lab as well as others revealed that $\mathrm{Cr}^{3+}$ exerts its influence on PM parameters [4-6]. Interestingly, these studies suggested that regulation of glucose transport by $\mathrm{Cr}^{3+}$ may be independent of amplification of insulin signaling, as previously described [7]. Rather, it was found that $\mathrm{Cr}^{3+}$ normalized elevated PM cholesterol levels that impaired glucose transport in 3T3-L1 adipocytes cultured under the diabetic milieu. Exogenous add back of cholesterol, for instance, blunted the effects of $\mathrm{Cr}^{3+}$ observed in these insulin-resistant cells. Despite this association in clonal cells, a basis for alterations in PM cholesterol content in promoting the development of insulin resistance remained unclear.

Parallel experiments have since established that nutriaent overabundance promotes elevations in membrane cholesterol content in 3T3-L1 adipocytes and L6 myotubes, as well as in skeletal muscle of $\mathrm{C} 57 \mathrm{Bl} / 6 \mathrm{~J}$ mice and Zucker rats [8-12]. These membrane perturbations were observed concomitant with a loss of cortical filamentousactin (F-actin) necessary for proper incorporation of the insulin sensitive glucose transporter GLUT4 into the PM. Furthermore, increased membrane cholesterol content was also correlated with diminished glucose disposal rates in humans [10]. Mechanistically, data revealed increased glucose flux through the hexosamine biosynthesis pathway (HBP) promoted elevated $O$-linked $N$-acetylglucosamine $(O$-GlcNAc) modification of specificity protein 1 (Sp1), leading to transcriptional activation of HMG-CoA 
reductase, the rate limiting enzyme in cholesterol synthesis [12]. This culminated in increased PM cholesterol content that perturbed $\mathrm{F}$-actin structure and impinged upon insulin action. Inhibition of the HBP or Sp1 binding to the DNA attenuated hyperinsulinemia-induced PM cholesterol accumulation, F-actin loss, and insulin resistance. Strikingly, these increases in cholesterol synthesis were independently found to impair cholesterol efflux processes that HBP inhibition also corrected [13].

Based on these studies finding PM stress compromised insulin action, we hypothesized that $\mathrm{Cr}^{3+}$ may potentially counter excessive HBP activity thereby inhibiting a transcriptional response leading to dysregulated cholesterol synthesis. We report herein that $\mathrm{Cr}^{3+}$ protects against hyperinsulinemia-induced increases in PM cholesterol content through inhibition of this pathway. These studies highlight a novel protective role of this micronutrient on glucose metabolism entailing the maintenance of optimal membrane cholesterol content.

\section{Materials and Methods}

\subsection{Cell Culture and Treatments}

Murine 3T3L1 adipocytes purchased from Dr. Howard Green were cultured as described previously [14]. Briefly, preadipocytes were cultured in Dulbecco's Modified Eagle Medium (DMEM) containing $25 \mathrm{mM}$ glucose and $10 \%$ bovine calf serum at $37^{\circ} \mathrm{C}$ at $10 \% \mathrm{CO}_{2}$. Confluent fibroblasts were induced to differentiate as previously described [8]. All studies were performed on adipocytes between 8 and 12 days post initiation of differentiation. For treatments, cells were left untreated or treated with CrPic (10 nM, $16 \mathrm{~h}$ Nutrition 21) in the presence or absence of hyperinsulinemia (500 pM, $12 \mathrm{~h}$ Sigma) in serum free DMEM. Acute insulin stimulations were performed with a $100 \mathrm{nM}$ dose during the last $5 \mathrm{~min}$ for signaling or $30 \mathrm{~min}$ for glucose transport analyses.

\subsection{Glucose Uptake}

Glucose uptake assays were performed as described previously [12]. Briefly, treated cells were incubated in a KRPH buffer (136 mM NaCl, 20 mM HEPES, pH 7.4, 5 $\mathrm{mM}$ sodium phosphate, $\mathrm{pH} 7.4,4.7 \mathrm{mM} \mathrm{KCl}, 1 \mathrm{mM}$ $\mathrm{MgSO}_{4}, 1 \mathrm{mM} \mathrm{CaCl}$ ) for $15 \mathrm{~min}$. Cells were then left unstimulated or stimulated with $100 \mathrm{nM}$ insulin for 30 min and exposed to $50 \mu \mathrm{M}$ 2-deoxyglucose (2-DG) containing $0.5 \mu \mathrm{Ci} 2-\left[{ }^{3} \mathrm{H}\right]$ deoxyglucose (Perkin Elmer) in the absence or presence of $20 \mu \mathrm{M}$ cytochalasin B. After $10 \mathrm{~min}$, uptake was terminated by aspiration and quenched with the addition of $1.0 \mathrm{ml}$ of $10 \mu \mathrm{M}$ cytochalasin $\mathrm{B}$. Cells were solubilized in $0.2 \mathrm{~N} \mathrm{NaOH}$ and $\left[{ }^{3} \mathrm{H}\right]$ was measured by liquid scintillation. Counts were normalized to total cellular protein, determined by the Bradford method.

\subsection{Protein Analyses}

Whole cell lysates were prepared following treatments as previously described [12]. $30-50 \mu \mathrm{g}$ of lysates were resolved and immunoblotted with antibodies to HMGR (Millipore), p-Akt (Genscript) Akt2 (Cell Signaling), pAkt substrate of 160 kilodaltons (Cell Signaling), and AS160 (Upstate). Immunoblots were quantitated using a LI-COR Odyssey infrared imaging system, normalizing signal intensity to $\beta$-actin (Cytoskeleton). For immunoprecipitation, $1.5 \mathrm{mg}$ of protein and $2 \mu \mathrm{g}$ of $\mathrm{Sp} 1$ antibody (Santa Cruz) were used as previously described [12]. Eluted immunoprecipitates were then resolved and immunoblotted using an RL2 antibody (Thermo Scientific).

\subsection{Cholesterol Measurements}

For cholesterol measurements, purified PM fractions were obtained as described [12] and cholesterol content was assayed using the Amplex Red cholesterol assay kit (Molecular Probes). Briefly, reconstituted PM pellets were vigorously mixed with chloroform-methanol (2:1 $\mathrm{v} / \mathrm{v}$ ) for $10 \mathrm{~min}$ to extract the cholesterol. The mixture was then centrifuged at $1000 \mathrm{rpm}$ for $10 \mathrm{~min}$. The lower phase containing lipids was then evaporated at $100^{\circ} \mathrm{C}$ for $10 \mathrm{~min}$. The residue was reconstituted with isopropranol-Triton X solution (10:1 v/v) and $50 \mu$ l of sample was combined with $50 \mu$ lof Amplex Red reaction mix and incubated at $37^{\circ} \mathrm{C}$ for $30 \mathrm{~min}$. After incubation, absorbance was measured at $600 \mathrm{~nm}$.

\subsection{Chromatin Immunoprecipitation}

Chromatin immunoprecipitation was performed on treated adipocytes as described previously [12]. Briefly, adipocytes were fixed with $1 \%$ formaldehyde for $10 \mathrm{~min}$. Cells were then scraped in PBS plus protease inhibitor cocktail and centrifuged for $2 \mathrm{~min}$ at $2000 \mathrm{rpm}$. The pellet was resuspended in $500 \mu \mathrm{l} \mathrm{ChIP} \mathrm{lysis} \mathrm{buffer}(50 \mathrm{mM}$ Tris $\mathrm{pH} 8.1,10 \mathrm{mM}$ EDTA, $1 \%$ SDS plus protease inhibitor cocktail), sonicated (10 pulses of $30 \mathrm{~s}$ on, $30 \mathrm{~s}$ off), and centrifuged at $14,000 \mathrm{rpm}$ at $4^{\circ} \mathrm{C}$ for $10 \mathrm{~min}$. Fragmented chromatin preparations were then diluted with ChIP dilution buffer (Millipore), and an input sample was collected. Samples were precleared prior to immunoprecipitation with antibodies to Sp1 (Santa Cruz) or IgG (Millipore) overnight. Eluates were reversed crosslinked and purified DNA was recovered using the phenol/chloroform method. DNA was subsequently used as a template for qPCR under the following conditions: 15 min at $95^{\circ} \mathrm{C}$ followed by 35 cycles of $15 \mathrm{~s}$ at $95^{\circ} \mathrm{C}, 30 \mathrm{~s}$ at $50^{\circ} \mathrm{C}$, and $34 \mathrm{~s}$ at $60^{\circ} \mathrm{C}$. The $\%$ input method was used to quantitate cycle threshold $(\mathrm{Ct})$ values. The sequence of 
the forward primer was (5'-3') ACCCGTCATTGGTTGGCTCT and reverse primer (5'-3') CTCCCTAACAACCGCCAACT.

\subsection{Plasmids and Luciferase Assays}

The proximal promoter sequence of HMGR ( -284 to +36 ), required for high level expression, was amplified from mouse genomic DNA using PCR $[15,16]$. Promoter fragments were sequenced and cloned into pGL2B luciferase reporter plasmids (Promega, Madison WI). Differentiated adipocytes were electroporated $(0.16 \mathrm{kV}$ and $960 \mu \mathrm{F})$ as previously described [12]. Briefly, cells were trypsinized and pelleted by centrifugation at $1000 \mathrm{rpm}$. Pellets were resuspended in PBS and centrifuged. Pellets were then resuspended in $0.5 \mathrm{ml}$ PBS. For transfection, $50 \mu \mathrm{g}$ of HMGR pGL ${ }_{2} \mathrm{~B}$ and $50 \mu \mathrm{g}$ of phrlmin TK (Renilla) plasmid were used with a concentration of approximately $1 \times 10^{7}$ cells $/ 0.5 \mathrm{ml}$. A single pulse was applied using a Gene Pulser (Bio-Rad \#1652076). The electroporated cells were then allowed to recover and plated into a 24 well plate. Treatments were begun $24 \mathrm{~h}$ after electroporation. After treatments, cells were lysed and assayed for promoter activity using the dualluciferase reporter assay system (Promega, Madison WI). Firefly Luciferase activities were normalized to Renilla activity to control for differences in transfection efficiency.

\subsection{RNA Analyses}

Adipocyte RNA was isolated using an RNeasy mini kit (Qiagen) according to the manufacturer's protocols. RNA was reverse transcribed using a High Capacity cDNA Reverse Transcription Kit (Applied Biosystems). Subsequent qPCR reactions were performed under the following conditions: $15 \mathrm{~min}$ at $95^{\circ} \mathrm{C}$ followed by 40 cycles of $15 \mathrm{~s}$ at $95^{\circ} \mathrm{C}$ and $40 \mathrm{~s}$ at $60^{\circ} \mathrm{C}$. Ct values were normalized to $36 \mathrm{~B} 4$. The $\Delta \Delta \mathrm{Ct}$ method was used to determine relative expression levels. The sequence for the HMGR forward primer was (5'-3') TGTGGGAACGGTGACACTTA and reverse primer (5'-3') CTTCAAATTTTGGGCACTCA. The sequence of for the 36B4 forward primer was (5'-3') AAGCGCGTCCTGGCATTGTCT and reverse primer (5'-3') CCGCAGGGGCAGCAGTGGT.

\subsection{Statistical Analyses}

Values presented are means \pm SEM. The significance of difference between means was evaluated by repeated measures ANOVA. Where a difference was indicated by ANOVA, a Newman-Keuls post-hoc test was used to compare differences between groups. Statistical comparisons of the fold or percent change of HMGR expression and Sp1 O-linked glycosylation were performed by two- tailed Student's $t$ test analysis. GraphPad Prism 5 software was used for all analyses. $\mathrm{P}<0.05$ was considered significant.

\section{Results}

\subsection{CrPic Prevents Impaired Glucose Transport}

Study first sought to examine the beneficial effects of $\mathrm{CrPic}$ treatment on cells rendered insulin resistant. Low doses of pathophysiologic hyperinsulinemia, utilized by many groups to induce insulin resistance [17-20], resulted in a $50 \%$ reduction in the ability of a maximal dose of insulin to stimulate glucose transport into adipocytes (Figure 1). In cells treated with hyperinsulinemia in the presence of a low, pharmacologically relevant dose $(10 \mathrm{nM})$ of CrPic for $16 \mathrm{~h}[4,6]$, the acute ability of insulin to stimulate glucose transport was corrected. Studies have suggested that a beneficial aspect of $\mathrm{CrPic}$ on glucose transport may entail countering defects in insulin signaling, thereby improving GLUT4 translocation to the PM [21-24]. Importantly, cell systems that mimic pathophysiological hyperinsulinemia, known to promote the progression/worsening of insulin resistance, and $\mathrm{CrPic}$, known to improve insulin action, are not associated with insulin signaling changes $[4,6,11,17]$. We thus next sought to verify that insulin signaling was indeed intact under hyperinsulinemic conditions and that the beneficial effect of CrPic was not coupled to enhancement in insulin signaling in these cells. Neither hyperinsulinemia, nor CrPic had any effects on signaling to Akt or its downstream target, AS160. Combinatorial treatment also did not affect any signaling parameters examined (Figure 2).

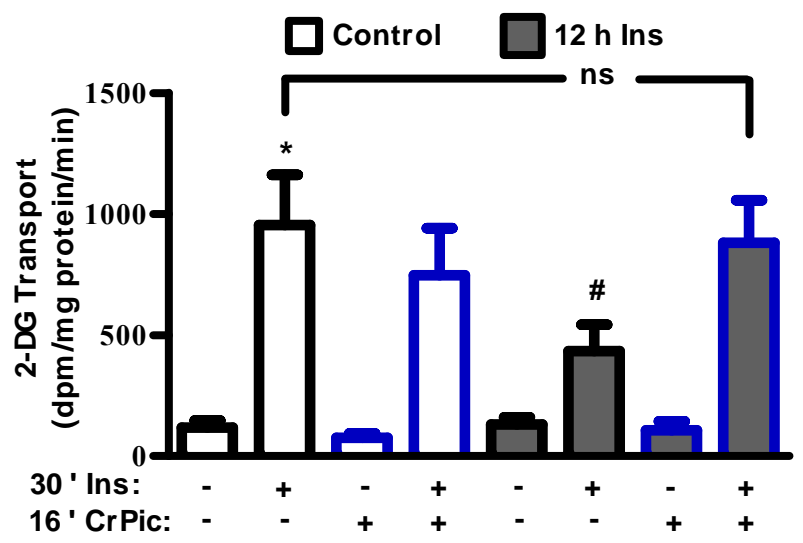

Figure 1. CrPic improves insulin responsiveness rendered impaired by hyperinsulinemia. 3T3-L1 adipocytes were treated with or without $10 \mathrm{nM}$ CrPic for $16 \mathrm{~h}$ and $500 \mathrm{pM}$ insulin for $12 \mathrm{~h}$. After treatments, 3T3-L1 adipocytes were left unstimulated or stimulated by a maximal dose (100 $\mathrm{nM})$ of insulin for $30 \mathrm{~min}$ to initiate glucose uptake. Values are mean \pm SEM from 4 - 6 independent experiments. ${ }^{*} P<0.05$ versus unstimulated control; ${ }^{\#} P<0.05$ versus all other 30' insulin groups; ns, non-significant. 


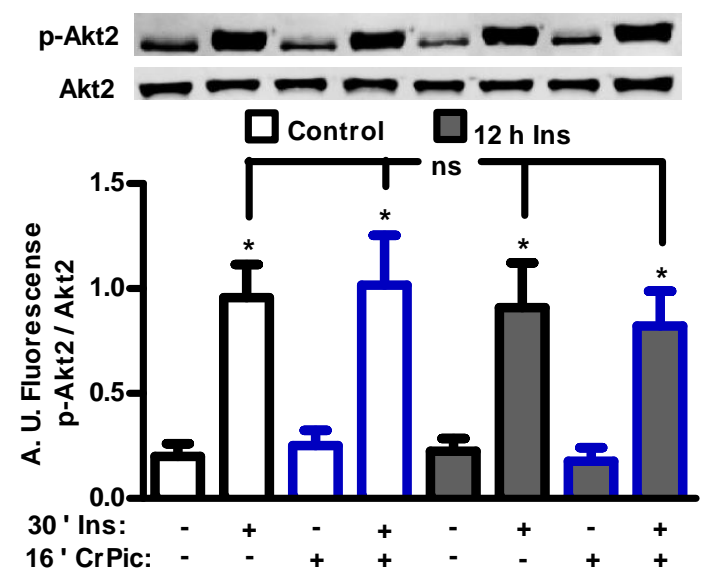

(a)

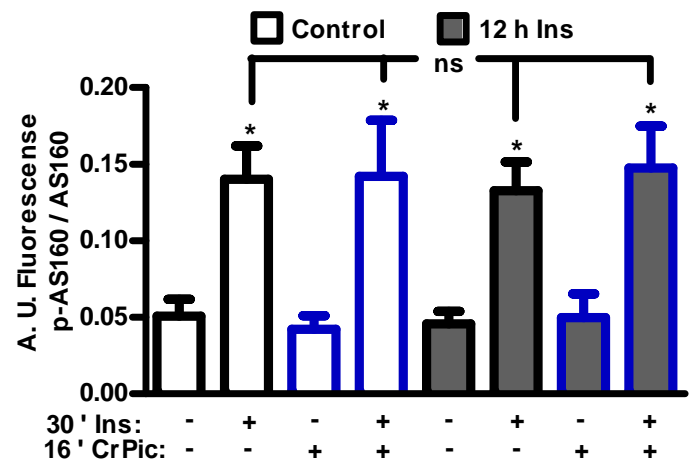

(b)

Figure 2. CrPic does not enhance nor does hyperinsulinemia impair insulin signaling. After treatments, 3T3-L1 adipocytes were left unstimulated or stimulated by a maximal dose (100 $\mathrm{nM})$ of insulin for 5 minutes to induce phosphorylation of insulin signaling proteins. A, Phosphorylation of Akt2 (Ser 474) normalized to total Akt2. B, Phosphorylation of AS160 normalized to total AS160. Values are mean \pm SEM from 4 independent experiments. ${ }^{\text {* }} \mathrm{P}<0.05$ versus respective unstimulated cells; ns, non-significant.

\subsection{CrPic Inhibits PM Cholesterol Accumulation}

Studies have demonstrated that hyperinsulinemia promotes increased PM cholesterol, in turn perturbing cortical F-actin necessary for proper GLUT4 incorporation into the PM [8,9]. Ex vivo examination of skeletal muscle from insulin-resistant Zucker rats demonstrated that correction of membrane cholesterol restores actin structure and insulin sensitivity [9]. It was examined whether CrPic treatment had beneficial effects on PM cholesterol levels. PM cholesterol content was elevated by approximately $50 \%$ in adipocytes exposed to hyperinsulinemia (Figure 3). Importantly, concurrent treatment with $\mathrm{CrPic}$ prevented this gain in cholesterol content.

\subsection{CrPic Prevents $O$-GIcNAcylation of Sp1}

Recent findings have established excessive glucose flux

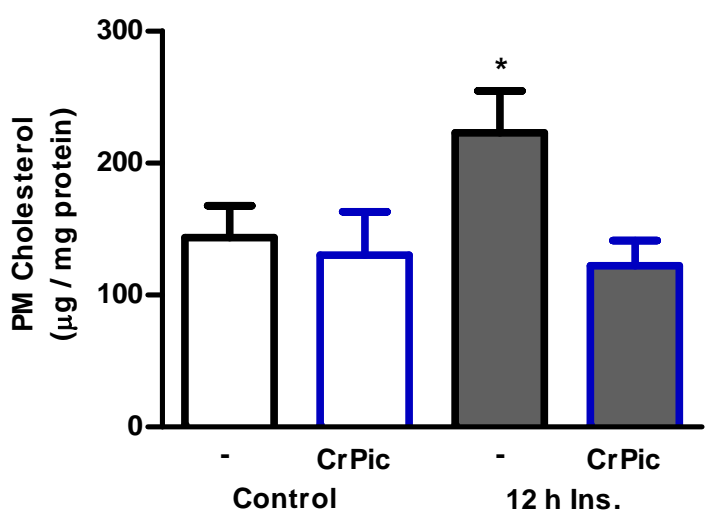

Figure 3. CrPic protects against PM cholesterol accumulation induced by hyperinsulinemia. After treatments, 3T3-L1 adipocytes were lysed and fractionated to prepare purified PM fractions. Cholesterol content in PM fractions was assessed via Amplex Red, normalized to PM protein content. Values are mean \pm SEM from 5 independent experiments. ${ }^{*} \mathbf{P}<\mathbf{0 . 0 5}$ versus all other groups.

through the HBP in provoking a cholesterolgenic response through modification of Sp1 [12]. To test whether CrPic could be potentiating HBP activity, thereby reducing a transcriptional response leading to increased PM cholesterol, the glycosylation status of Sp1 was next examined. These analyses revealed both a gain in global $O$ GlcNAc levels (data not shown) as well as increased $O$ GlcNAc of Sp1 induced by hyperinsulinemia (Figure 4). While CrPic treatment did not alter HBP activity in control cells, it blunted the effects of hyperinsulinemia in engaging the HBP.

\subsection{CrPic Attenuates Sp1 Affinity to and Activity of the HMGR Promoter}

Study next sought to analyze whether CrPic may promote optimal glucose transport by inhibiting a HBP-induced cholesterolgenic transcriptional response entailing $\mathrm{Sp} 1$. In untreated cells cultured in low or high glucose, a majority of Sp1 is localized to the nucleus [25], although some studies suggest $O$-GlcNAc mediates its nuclear localization [26,27]. Other data, including our own, suggest that $O$-GlcNAc modification may serve to increase Sp1 binding to the promoter region of target genes or prevent its degradation [12,28,29]. Chromatin immuno-precipitation revealed a significant increase in Sp1 binding affinity to the HMGR promoter induced by hyperinsulinemia, whereas treatment with $\mathrm{CrPic}$ blunted this association (Figure 5). To discern if $\mathrm{CrPic}$ could inhibit activation the promoter, plasmids containing the coding sequence ( -284 to +36$)$ of HMGR, including $3 \mathrm{Sp} 1$ binding moieties, coupled to luciferase, were electroporated into 3T3L1 adipocytes (Figure 6(a)). Luciferase activity was elevated approximately 2 fold by hyperinsulinemia (Figure 6(b)). Consistent with $\mathrm{CrPic}$ inhibiting this response, 


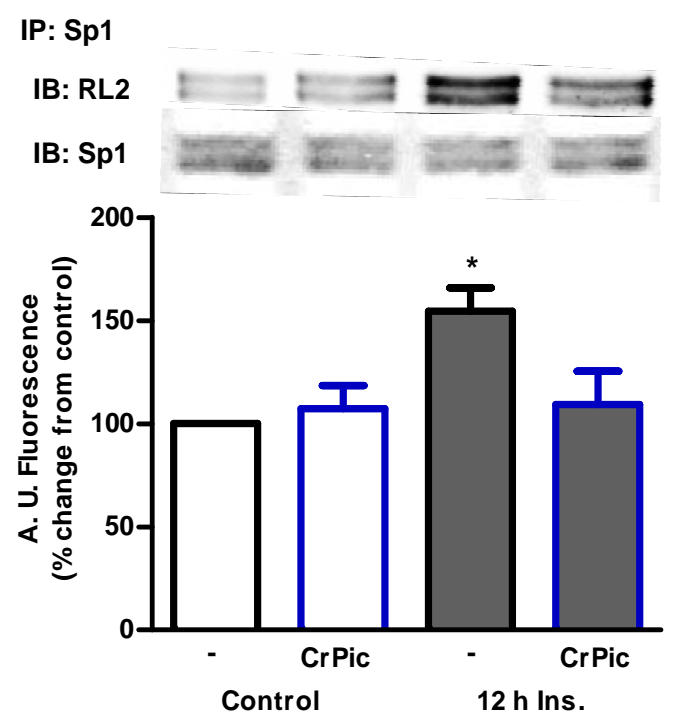

Figure 4. Hyperinsulinemia provokes and CrPic inhibits O-GIcNAc of Sp1. After treatments, lysates were immunoprecipitated with a $\mathrm{Sp1}$ antibody. Eluted samples were subsequently immunoblotted and labeled with an RL2 antibody to detect $O$-GIcNAc on Sp1. Values represent the mean \pm SEM from 5 independent experiments. " $P<0.05$ versus untreated control.

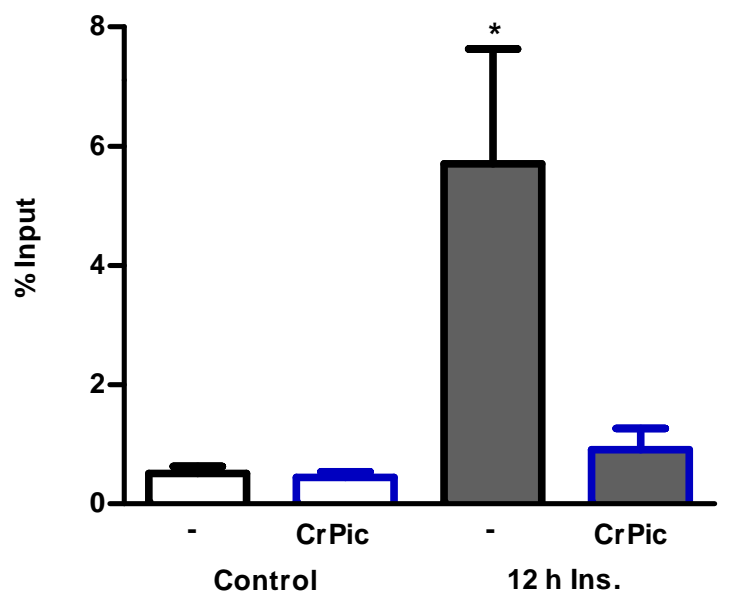

Figure 5. CrPic reduced hyperinsulinemia-induced association of Sp1 toward the HMGR promoter. After treatments, ChIP was performed and primers specific to the Sp1 binding site in the promoter region of HMGR were used for amplification of the DNA eluates via qPCR. Ct values from qPCR were normalized using the fold enrichment method. Values are mean \pm SEM from 3 independent experiments. ${ }^{*} \mathbf{P}<\mathbf{0 . 0 5}$ versus untreated control.

elevations in luciferase activity were not observed in cells treated with CrPic.

\subsection{CrPic Inhibits HMGR Synthesis}

It was next examined whether $\mathrm{CrPic}$, through attenuating Sp1 affinity to the promoter region of HMGR, as well as activity of the promoter, could inhibit HMGR synthesis
-284 catcccetgttccecqcqcqgacqqcqtccttgcagge

-246 gecctcggetcggagaccaataggaaggccgcgatg

-209 ctgggaccogacccqtcattgattgqctctgecqtqqutga

C/EBP

SRE

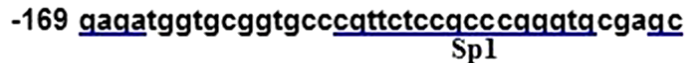

-131 agtqgacqqttqttagggagaccqttcqtgacqtaggec Spl

CRE

-92 gtcaggctgagcagccgeccqacqattqgctaqgggat C/EBP -54 cggacgatccttcettattgqcquccegetggeggectg

-15 gagcgtgcgtaagcgcagttccttccgcceggggetcc $+1$

+23 gttggctggagacatg $\frac{a 8}{*}$

(a)

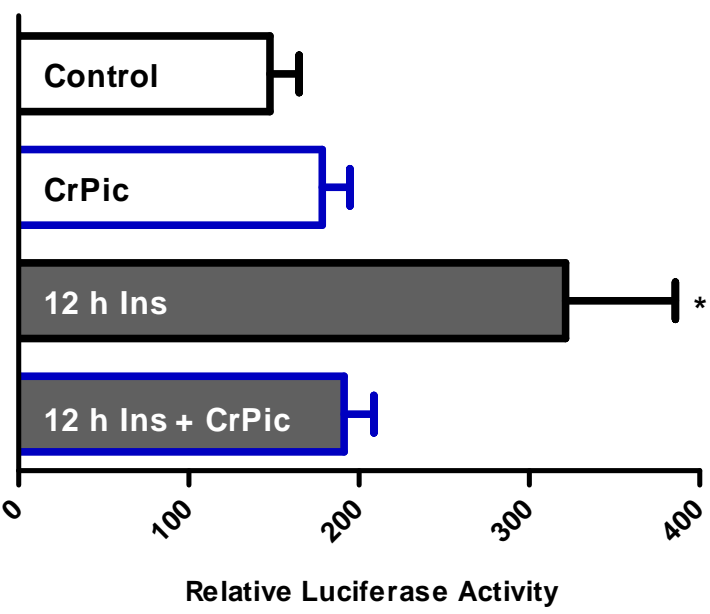

(b)

Figure 6. Hyperinsulinemia activates and $\mathrm{CrPic}$ reduces the promoter activity of HMGR. A, the nucleotide sequence of the minimal HMGR promoter $(-284 /+38)$ is shown. The location of various sequence motifs that serve as sites of recognition for transcription factors are underlined including those for Sp1 (C/EBP, CCAAT/enhancer binding protein SRE, sterol regulatory element, CRE, cAMP response element). The +1 indicates the start site of transcription and the start site of translation is indicated by an asterisk. $B$, Hmger pGL $_{2} B$ and phrl-minTK constructs were transfected into 3T3-L1 adipocytes. After transfection, cells were left untreated or treated with $500 \mathrm{pM}$ insulin and/or $10 \mathrm{nM}$ CrPic. Cells were then lysed in passive lysis buffer and luciferase activity was measured, normalized to Renilla. Values are mean \pm SEM from 3 independent experiments. " $\mathrm{P}<$ 0.05 versus control.

as this enzyme is rate-limiting in the formation of cholesterol. Hyperinsulinemia was found to increase HMGR mRNA by approximately 5 fold, whereas $\mathrm{CrPic}$ prevented this alteration (Figure 7(a)). Strikingly, CrPic treatment also attenuated a $60 \%$ gain in protein content of HMGR that was observed in hyperinsulinemic cells 
(Figure 7(b)). Together, these data suggest a transcriptional basis for the beneficial effects of CrPic on glucose transport processes.

\section{Discussion}

The current studies provide novel mechanistic insight into the established role of $\mathrm{CrPic}$ in benefiting glucose homeostasis. Data support a transcriptional basis for CrPic action in preventing PM cholesterol accumulation, which has been previously shown to impinge upon insulin action. While the mechanisms by which CrPic may precisely inhibit glucose flux through and/or activity of the HBP were not a focus of this work, study has demonstrated that CrPic activates AMPK, a known sensor of

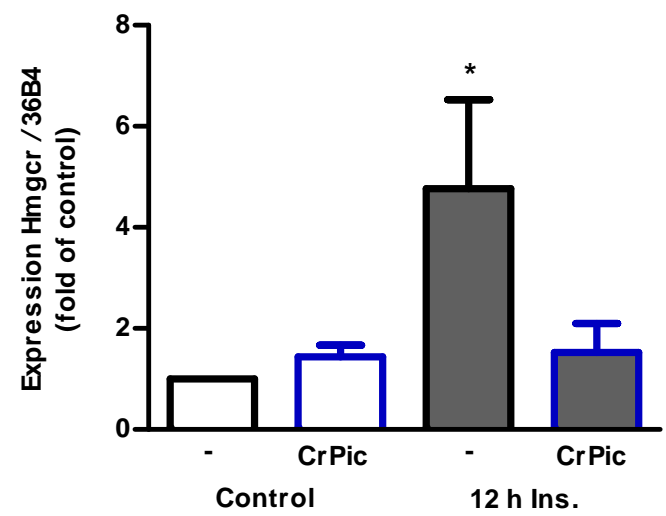

(a)

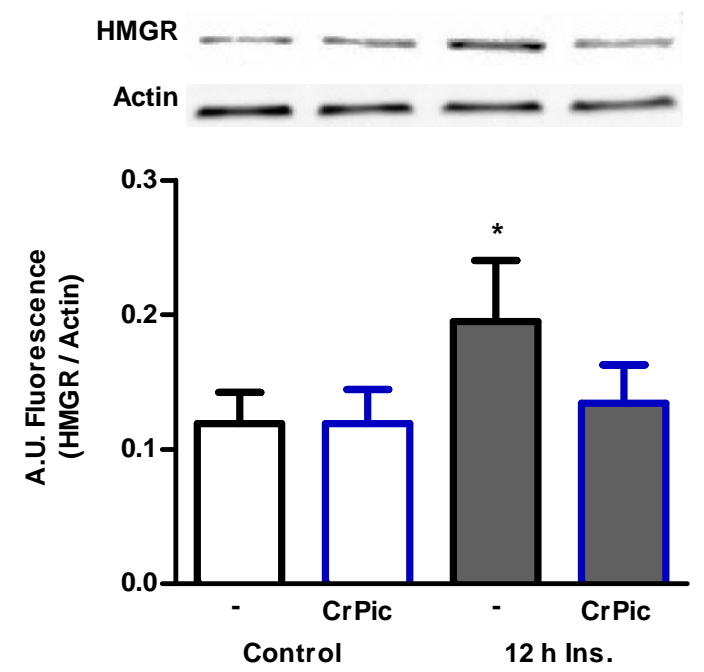

(b)

Figure 7. CrPic protects against hyperinsulinemia-induced HMGR synthesis. A, After treatments, RNA was purified, reverse transcribed, and HMGR mRNA expression levels were assessed via qPCR and quantitated using the $\Delta \Delta \mathrm{ct}$ method, normalized to 36B4 mRNA expression. B, After treatments HMGR protein content was assessed via western blotting, normalized to actin. Values are mean \pm SEM from 4 - 5 independent experiments. ${ }^{*} P<0.05$ versus untreated control. low energy status $[4,6,13,30,31]$. Additionally, it has recently been determined that AMPK can phosphorylate and inhibit glutamine fructose-6-phosphate amidotransferase, the rate limiting enzyme in the HBP [32]. In this regard, CrPic may have pleiotropic effects on cholesterol synthesis processes through inhibiting both production of HMGR as well as its activity.

The exact mechanisms whereby this micronutrient may trigger the activation of AMPK to potentially modulate flux through this pathway are thus warranted and may involve liver kinase B1, calmodulin activated protein kinase kinases, protein tyrosine phosphatase $2 \mathrm{~A}$ and/ or alterations in the cellular ATP/AMP ratio through altering mitochondrial complexes [33-36].

With regard to the HBP, approximately $25 \%$ of proteins modified by $O$-GlcNAc are transcription factors [37]. Sp1 was the primary focus of this work due to recent study suggesting inhibition of Sp1 could protect against glucose transport dysfunction in adipocytes [12]. Nevertheless, HBP-induced $O$-GlcNAc on histones has been demonstrated [38]. Interestingly, this study found that many metabolic gene products, including HMGR, were upregulated in response to activation of the HBP. As HBP-induced modification of proteins is known to occur in times of nutrient excess, global inhibition of this pathway by $\mathrm{CrPic}$ is of interest as it may have beneficial metabolic effects extending beyond maintenance of cholesterol synthesis. In this regard, a study has shown that $\mathrm{CrPic}$ or HBP inhibition restores cholesterol efflux rendered impaired by hyperinsulinemia [13]. While the current study focused on inhibition of cholesterol synthesis, $\mathrm{CrPic}$ has been documented to be necessary for maintenance or even improve high density lipoprotein levels in humans given brewer's yeast $[39,40]$. Together, these findings support a mechanism whereby CrPic may benefit glucose metabolism by maintaining optimal PM cholesterol levels needed for appropriate glucose transport into peripheral tissues.

In terms of human health, our data suggest a novel, putative mechanism whereby $\mathrm{CrPic}$ could be beneficial to glucose metabolism through countering PM stress. While clinical trials suggest a beneficial effect of $\mathrm{CrPic}$ in diabetics, it may be possible that CrPic treatment has an effect to counter dysregulation in membrane stress and that this incremental effect may become impeded later in disease progression when other factors further perturb insulin sensitivity. Alternatively, antihyperglycemic medications could mask the effects of CrPic, as many are known to activate AMPK. Further, well-designed longitudinal studies are thus needed in insulin-resistant, nondiabetic patients to help better characterize the role of this micronutrient in alleviating insulin resistance. In this regard, CrPic use may prove beneficial in preventing the exacerbation of insulin resistance in patient populations 
through promoting optimal PM fluidity.

\section{Acknowledgements}

This work was supported by grants from the National Institutes of Health (DK082773) as well as an Indiana University School of Medicine Moenkhaus Endowment.

\section{REFERENCES}

[1] R. A. Anderson, et al., "Elevated Intakes of Supplemental Chromium Improve Glucose and Insulin Variables in Individuals with Type 2 Diabetes," Diabetes, Vol. 46, No. 11, 1997, pp. 1786-1791. doi:10.2337/diabetes.46.11.1786

[2] E. M. Balk, A. Tatsioni, A. H. Lichtenstein, J. Lau and A. G. Pittas, "Effect of Chromium Supplementation on Glucose Metabolism and Lipids: A Systematic Review of Randomized Controlled Trials," Diabetes Care, Vol. 30, No. 8, 2007, pp. 2154-2163. doi:10.2337/dc06-0996

[3] S. R. Surani, I. Ratnani, B. Guntupalli and S. Bopparaju, "Severe Insulin Resistance Treatment with Intravenous Chromium in Septic Shock Patient," World Journal of Diabetes, Vol. 3, No. 9, 2012, pp. 170-173. doi:10.4239/wjd.v3.19.170

[4] Chen, G., et al., "Chromium Activates Glucose Transporter 4 Trafficking and Enhances Insulin-Stimulated Glucose Transport in 3T3-L1 Adipocytes via a CholesterolDependent Mechanism," Molecular Endocrinology, Vol. 20, No. 4, 2006, pp. 857-870. doi:10.1210/me.2005-0255

[5] G. W. Evans and T. D. Bowman, "Chromium Picolinate Increases Membrane Fluidity and Rate of Insulin Internalization," Journal of Inorganic Biochemistry, Vol. 46, No. 4, 1992, pp. 243-250. doi:10.1016/0162-0134(92)80034-S

[6] G. R. Pattar, L. Tackett, P. Liu and J. S. Elmendorf, "Chromium Picolinate Positively Influences the Glucose Transporter System via Affecting Cholesterol Homeostasis in Adipocytes Cultured under Hyperglycemic Diabetic Conditions," Mutation Research, Vol. 610, No. 1-2, 2006, pp. 93-100. doi:10.1016/j.mrgentox.2006.06.018

[7] D. L. Brautigan, A. Kruszewski and H. Wang, "Chromium and Vanadate Combination Increases Insulin-Induced Glucose Uptake by 3T3-L1 Adipocytes," Biochemical and Biophysical Research Communications, Vol. 347, No. 3, 2006, pp. 769-773. doi:10.1016/j.bbrc.2006.06.154

[8] P. Bhonagiri, et al., "Evidence Coupling Increased Hexosamine Biosynthesis Pathway Activity to Membrane Cholesterol Toxicity and Cortical Filamentous Actin Derangement Contributing to Cellular Insulin Resistance," Endocrinology, Vol. 152, No. 9, 2011, pp. 3373-3384. doi:10.1210/en.2011-1295

[9] K. M. Habegger, N. J. Hoffman, C. M. Ridenour, J. T. Brozinick and J. S. Elmendorf, "AMPK Enhances Insulin-Stimulated GLUT4 Regulation via Lowering Membrane Cholesterol," Endocrinology, Vol. 153, No. 5, 2012, pp. 2130-2141. doi:10.1210/en.2011-2099

[10] K. M. Habegger, et al., "Fat-Induced Membrane Cholesterol Accrual Provokes Cortical Filamentous Actin Desta- bilisation and Glucose Transport Dysfunction in Skeletal Muscle," Diabetologia, Vol. 55, No. 2, 2012, pp. 457-467. doi:10.1007/s00125-011-2334-y

[11] A. M. McCarthy, K. O. Spisak, J. T. Brozinick and J. S. Elmendorf, "Loss of Cortical Actin Filaments in InsulinResistant Skeletal Muscle Cells Impairs GLUT4 Vesicle Trafficking and Glucose Transport," American Journal of Physiology Cell Physiology, Vol. 291, No. 5, 2006, pp. C860-C868. doi:10.1152/ajpcell.00107.2006

[12] B. A. Penque, A. M. Hoggatt, B. P. Herring and J. S. Elmendorf, "Hexosamine Biosynthesis Impairs Insulin Action via a Cholesterolgenic Response," Molecular Endocrinology, 2013. doi:10.1210/me.2012-1213

[13] W. Sealls, B. A. Penque and J. S. Elmendorf, "Evidence That Chromium Modulates Cellular Cholesterol Homeostasis and ABCA1 Functionality Impaired by Hyperinsulinemia-Brief Report," Arteriosclerosis, Thrombosis, and Vascular Biology, Vol. 31, No. 5, 2011, pp. 1139-1140. doi:10.1161/ATVBAHA.110.222158

[14] H. Green and M. Meuth, "An Established Pre-Adipose Cell Line and Its Differentiation in Culture," Cell, Vol. 3, No. 2, 1974, pp. 127-133. doi:10.1016/0092-8674(74)90116-0

[15] T. F. Osborne, G. Gil, M. S. Brown, R. C. Kowal and J. L. Goldstein, "Identification of Promoter Elements Required for in Vitro Transcription of Hamster 3-Hydroxy-3-methylglutaryl Coenzyme A Reductase Gene," Proceedings of National Academy of Science of USA, Vol. 84, No. 11, 1987, pp. 3614-3618. doi:10.1073/pnas.84.11.3614

[16] T. F. Osborne, J. L. Goldstein and M. S. Brown, "5' End of HMG CoA Reductase Gene Contains Sequences Responsible for Cholesterol-Mediated Inhibition of Transcription," Cell, Vol. 42, No. 1, 1985, pp. 203-212. doi:10.1016/S0092-8674(85)80116-1

[17] G. Chen, et al., "Protective Effect of Phosphatidylinositol 4,5-Bisphosphate against Cortical Filamentous Actin Loss and Insulin Resistance Induced by Sustained Exposure of 3T3-L1 ADipocytes to Insulin," The Journal of Biological Chemistry, Vol. 279, No. 38, 2004, pp. 39705-39709. doi:10.1074/jbc.C400171200

[18] Y. Ng, G. Ramm and D. E. James, "Dissecting the Mechanism of Insulin Resistance Using a Novel Heterodimerization Strategy to Activate Akt," The Journal of Biological Chemistry, Vol. 285, No. 8, 2010, pp. 5232-5239. doi:10.1074/jbc.M109.060632

[19] K. A. Robinson and M. G. Buse, "Mechanisms of HighGlucose/Insulin-Mediated Desensitization of Acute Insulin-Stimulated Glucose Transport and Akt Activation," American Journal of Physiology-Endocrinology and Metabolism, Vol. 294, No. 5, 2008, pp. E870-E881. doi:10.1152/ajpendo.00644.2007

[20] W. Xiong, I. Jordens, E. Gonzalez and T. E. McGraw, "GLUT4 Is Sorted to Vesicles Whose Accumulation Beneath and Insertion into the Plasma Membrane Are Differentially Regulated by Insulin and Selectively Affected by Insulin Resistance," Molecular Biology of the Cell, Vol. 21, No. 8, 2010, pp. 1375-1386. doi:10.1091/mbc.E09-08-0751

[21] W. T. Cefalu, Z. Q. Wang, X. H. Zhang, L. C. Baldor and J. 
C. Russell, "Oral Chromium Picolinate Improves Carbohydrate and Lipid Metabolism and Enhances Skeletal Muscle Glut-4 Translocation in Obese, Hyperinsulinemic (JCR-LA Corpulent) Rats," Journal of Nutrition, Vol. 132, No. 6, 2002, pp. 1107-1114.

[22] F. Dong, M. R. Kandadi, J. Ren and N. Sreejayan, "Chromium (D-phenylalanine)3 Supplementation Alters Glucose Disposal, Insulin Signaling, and Glucose Transporter-4 Membrane Translocation in Insulin-Resistant Mice," Journal of Nutrition, Vol. 138, No. 10, 2008, pp. 1846-1851.

[23] M. R. Kandadi, et al., "Chromium (D-Phenylalanine)3 Alleviates High Fat-Induced Insulin Resistance and Lipid Abnormalities," Journal of Inorganic Biochemistry, Vol. 105, No. 1, 2011, pp. 58-62. doi:10.1016/i.jinorgbio.2010.09.008

[24] Z. Q. Wang, X. H. Zhang, J. C. Russell, M. Hulver and W. T. Cefalu, "Chromium Picolinate Enhances Skeletal Muscle Cellular Insulin Signaling in Vivo in Obese, InsulinResistant JCR:LA-Cp Rats," Journal of Nutrition, Vol. 136, No. 2, 2006, pp. 415-420.

[25] H. J. Goldberg, C. I. Whiteside, G. W. Hart and I. G. Fantus, "Posttranslational, Reversible O-Glycosylation Is Stimulated by High Glucose and Mediates Plasminogen Activator Inhibitor-1 Gene Expression and Sp1 Transcriptional Activity in Glomerular Mesangial Cells," Endocrinology, Vol. 147, No. 1, 2006, pp. 222-231. doi:10.1210/en.2006-0523

[26] C. Brasse-Lagnel, A. Fairand, A. Lavoinne and A. Husson, "Glutamine Stimulates Argininosuccinate Synthetase Gene Expression through Cytosolic O-Glycosylation of Sp1 in Caco-2 Cells," The Journal of Biological Chemistry, Vol. 278, No. 52, 2003, pp. 52504-52510. doi:10.1074/jbc.M306752200

[27] G. Majumdar, et al., "Insulin Dynamically Regulates Calmodulin Gene Expression by Sequential O-Glycosylation and Phosphorylation of Sp1 and Its Subcellular Compartmentalization in Liver Cells," The Journal of Biological Chemistry, Vol. 281, No. 6, 2006, pp. 3642-3650. doi:10.1074/jbc.M511223200

[28] I. Han and J. E. Kudlow, "Reduced O Glycosylation of Sp1 Is Associated with Increased Proteasome Susceptibility," Molecular Cell Biology, Vol. 17, No. 5, 1997, pp. 25502558.

[29] C. Weigert, et al., "Palmitate-Induced Activation of the Hexosamine Pathway in Human Myotubes: Increased Expression of Glutamine: Fructose-6-Phosphate Aminotransferase," Diabetes, Vol. 52, No. 3, 2003, pp. 650-656. doi:10.2337/diabetes.52.3.650

[30] Y. Q. Wang, Y. Dong and M. H. Yao, "Chromium Picolinate Inhibits Resistin Secretion in Insulin-Resistant 3T3-
L1 Adipocytes via Activation of Amp-Activated Protein Kinase," Clinical and Experimental Pharmacology and Physiology, Vol. 36, No. 8, 2009, pp. 843-849. doi:10.1111/j.1440-1681.2009.05164.x

[31] P. Zhao, et al., "A Newly Synthetic Chromium ComplexChromium (D-Phenylalanine)3 Activates AMP-Activated Protein Kinase and Stimulates Glucose Transport," Biochemical Pharmacology, Vol. 77, No. 6, 2009, pp. $1002-$ 1010. doi:10.1016/j.bcp.2008.11.018

[32] S. Eguchi, et al., "AMP-Activated Protein Kinase Phosphorylates Glutamine: Fructose-6-phosphate Amidotransferase 1 at Ser243 to Modulate Its Enzymatic Activity," Genes Cells, Vol. 14, No. 2, 2009, pp. 179-189. doi:10.1111/j.1365-2443.2008.01260.x

[33] S. P. Davies, N. R. Helps, P. T. Cohen and D. G. Hardie, "5'-AMP Inhibits Dephosphorylation, as Well as Promoting Phosphorylation, of the AMP-Activated Protein Kinase. Studies Using Bacterially Expressed Human Protein Phosphatase-2C Alpha and Native Bovine Protein Phosphatase-2AC," FEBS Letters, Vol. 377, No. 3, 1995, pp. 421-425. doi:10.1016/0014-5793(95)01368-7

[34] D. G. Hardie, F. A. Ross and S. A. Hawley, "AMPK: A Nutrient and Energy Sensor That Maintains Energy Homeostasis," Nature Reviews Molecular Cell Biology, Vol. 13, No. 4, 2012, pp. 251-262. doi:10.1038/nrm3311

[35] J. S. Oakhill, et al., "AMPK Is A Direct Adenylate ChargeRegulated Protein Kinase," Science, Vol. 332, No. 6036, 2011, pp. 1433-1435. doi:10.1126/science. 1200094

[36] B. Xiao, et al., "Structure of Mammalian AMPK and Its Regulation by ADP," Nature, Vol. 472, No. 7342, 2011, pp. 230-233. doi:10.1038/nature09932

[37] D. C. Love and J. A. Hanover, "The Hexosamine Signaling Pathway: Deciphering the 'O-GlcNAc Code'," Science STKE, Vol. 2005, No. 312, 2005, p. re13.

[38] R. Fujiki, et al., "GlcNAcylation of Histone H2B Facilitates Its Monoubiquitination," Nature, Vol. 480, No. 7378, 2011, pp. 557-560.

[39] H. Khosravi-Boroujeni, A. Rostami, S. Ravanshad and A. Esmaillzadeh, "Favorable Effects on Metabolic Risk Factors with Daily Brewer's Yeast in Type 2 Diabetic Patients with Hypercholesterolemia: A Semi-Experimental Study," Journal of Diabetes, Vol. 4, No. 2, 2012, pp. 153158. doi:10.1111/j.1753-0407.2011.00163.x

[40] R. Riales and M. J. Albrink, "Effect of Chromium Chloride Supplementation on Glucose Tolerance and Serum Lipids including High-Density Lipoprotein of Adult Men," American Journal of Clinical Nutrition, Vol. 34, No. 12, 1981, pp. 2670-2678. 